\title{
The Bruhat Order on classes of isotopic Latin Squares
}

\author{
Rosário Fernandes*, Henrique F. da Cruz ${ }^{\dagger}$ Domingos Salomão
}

* CMA and Departamento de Matemática da Faculdade de Ciências e Tecnologia,

Universidade Nova de Lisboa, 2829-516 Caparica, Portugal.

$\dagger$ Universidade da Beira Interior, Centro de Matemática e Aplicações (CMA-UBI), Rua Marquês D'Ávila e Bolama, 6201-001 Covilhã, Portugal.

$\ddagger$ Centro de Matemática e Aplicações (CMA-UBI), Covilhã, and Universidade Mandume ya Ndemufayo, Escola Superior Pedagógica do Namibe, Angola,

June 22, 2020

\begin{abstract}
In a previous paper, the authors introduced and studied the Bruhat order in the class of Latin squares of order $n$. In this paper, we investigate the restriction of the Bruhat order in a class of isotopic Latin squares. We present equivalent conditions for two Latin squares be related by the Bruhat order when one of them is obtained from the other by interchanging rows or columns or symbols. The cover relation is also addressed, and we present orthogonal isotopic Latin squares related by the Bruhat order.
\end{abstract}

Keywords: Latin squares, Bruhat order, orthogonal Latin squares, transversals.

AMS Subject of Classification: 05B20, 05B15, 06A07

${ }^{*}$ e-mail adress: mrff@fct.unl.pt. This work was partially supported by the Fundação para a Ciência e a Tecnologia through the project UIBD/MAT/00297/2020.

$\dagger e$-mail address: hcruz@ubi.pt. This work was partially supported by the Fundação para a Ciência e a Tecnologia through the project UID/MAT/00212/2019.

${ }^{\ddagger}$ e-mail address: d.salomao@ubi.pt. 


\section{Introduction}

A Latin square of order $n$ is an $n$-by- $n$ grid filled with $n$ symbols so that each symbol appears once in each row and in each column. We use the integers $1, \ldots, n$ for the symbols. Latin squares have a long history, and their applications can be found in many areas. In cryptography, the multiplication table of a finite group or of a quasigroup are Latin squares that encode features of algebraic structures.

One notion very important in the study of Latin squares is the notion of transversal. A transversal of a Latin square is a list of $n$ distinct symbols, one from each row and each column. The question regarding the existence of a transversal in Latin squares that encode the Cayley tables of finite groups is an area of active investigation, $[1,3,20,21]$.

Let $A=\left[a_{i j}\right]$ and $B=\left[b_{i j}\right]$ be two Latin squares of order $n$. We say that $A$ is orthogonal to $B$ if

$$
\left\{\left(a_{i j}, b_{i j}\right):(i, j) \in\{1, \ldots, n\} \times\{1, \ldots, n\}\right\}=\{1, \ldots, n\} \times\{1, \ldots, n\} .
$$

It is known that if a Latin square $A$ is orthogonal to another Latin square then $A$ has, at least, $n$ transversals. In [1] the author proved that if $A$ is a Latin square of even order then $A$ has an even number of transversals and a stronger version of the theorem from [1] appears in [2]. If $A$ is a Latin square of odd order, Ryser, in [21], conjectured that $A$ has, at least, one transversal. This conjecture remains unproven in general, but relatively recent, aided by a computer, the authors of [20] proved that the conjecture holds for Latin squares of order less than 10 .

The Bruhat order is a partial order relation firstly defined on permutation matrices, but latter extended, by Brualdi and Hwang, to matrices whose all entries are zeros and ones, the $(0,1)$-matrices, [4]. If $A=\left[a_{i j}\right]$ and $C$ are two $m$-by- $n(0,1)$-matrices, then $A$ precedes $C$ by the Bruhat order, and we write $A \preceq_{B} C$, if by the entrywise order, $\Sigma(A) \geq \Sigma(C)$, where $\Sigma(A)=\left[\sigma_{r s}(A)\right]$ is the $m$-by- $n$ matrix whose $(r, s)$-entry is

$$
\sigma_{r, s}(A)=\sum_{i=1}^{r} \sum_{j=1}^{s} a_{i j}, \text { for } 1 \leq r \leq m, 1 \leq s \leq n,
$$

and $\Sigma(C)$ is defined similarly.

The Bruhat order on $(0,1)$-matrices is receiving the attention of many researchers, $[4,6,10,11,12,13,15,16,17,18,19]$. In the recent years several authors have taken Brualdi and Hwang's ideas, and extended the Bruhat order to other classes of matrices than $(0,1)$-matrices: the Bruhat order has been studied on the class of tournament matrices with a given score vector, [8], on the class of alternating sign matrices, [9], and on the class of doubly stochastic matrices, [7]. In [14], the authors, followed this new branch of investigation, studied the Bruhat order on the class of Latin squares of order $n$. This partial 
order relation is defined similarly as it is defined on other classes of matrices, that is, by using the matrices $\Sigma($.$) defined above.$

Let $A$ and $C=\left[c_{i j}\right]$ be two Latin squares of order $n$. If $\alpha$ is a permutation of $\{1, \ldots, n\}$, this is, $\alpha \in S_{n}$, we denote by $\alpha(C)$ the Latin square of order $n$ whose $(i, j)$ entry is $\alpha\left(c_{i j}\right)$. We say that $A$ and $C$ are isotopic if one can be turned into the other by permuting rows, columns and symbols, this is, there are permutation matrices $R, Q$, and a permutation $\alpha \in S_{n}$ such that $R A Q=\alpha(C)$.

Note that, $R A Q=\alpha(C)$ is equivalent to $\alpha^{-1}(R A Q)=C$. Sometimes we will write the permutations associated with the permutation matrices $R$ and $Q$, $\pi$ and $\rho$, respectively, instead of the matrices. So, $R$ and $Q$ will be written by $P(\pi)$ and $P(\rho)$, respectively. Moreover, the entry $(i, j)$ of $P(\pi)$ is

$$
P(\pi)_{i j}=\left\{\begin{array}{ll}
1 & \text { if } \pi(j)=i \\
0 & \text { otherwise }
\end{array},\right.
$$

similarly we define the entry $(i, j)$ of $P(\rho)$.

For instance, let

$$
A=\left[\begin{array}{llll}
1 & 2 & 3 & 4 \\
2 & 1 & 4 & 3 \\
3 & 4 & 1 & 2 \\
4 & 3 & 2 & 1
\end{array}\right] \quad \text { and } \quad D=\left[\begin{array}{cccc}
3 & 2 & 1 & 4 \\
1 & 4 & 3 & 2 \\
2 & 3 & 4 & 1 \\
4 & 1 & 2 & 3
\end{array}\right]
$$

If $R$ is the permutation matrix associated with the permutation $\pi=(23)$ and, $\alpha=(13)$ then

$$
R A=\left[\begin{array}{llll}
1 & 2 & 3 & 4 \\
3 & 4 & 1 & 2 \\
2 & 1 & 4 & 3 \\
4 & 3 & 2 & 1
\end{array}\right]=\left[\begin{array}{llll}
\alpha(3) & \alpha(2) & \alpha(1) & \alpha(4) \\
\alpha(1) & \alpha(4) & \alpha(3) & \alpha(2) \\
\alpha(2) & \alpha(3) & \alpha(4) & \alpha(1) \\
\alpha(4) & \alpha(1) & \alpha(2) & \alpha(3)
\end{array}\right]=\alpha(D)
$$

So, $A$ and $D$ are isotopic Latin squares.

Let

$$
A=\left[\begin{array}{llll}
1 & 2 & 3 & 4 \\
2 & 1 & 4 & 3 \\
3 & 4 & 1 & 2 \\
4 & 3 & 2 & 1
\end{array}\right] \quad \text { and } \quad C=\left[\begin{array}{llll}
1 & 2 & 3 & 4 \\
2 & 1 & 4 & 3 \\
3 & 4 & 2 & 1 \\
4 & 3 & 1 & 2
\end{array}\right]
$$

Then $A$ and $C$ are not isotopic. In fact, in the first row of $A$, the symbol 1 appears before 2 and the symbol 3 before 4 , and in all other rows of $A$ we have

1 before 2 , and 3 before 4 ,

or

2 before 1 , and 4 before 3 .

When we interchange the rows, the columns and the symbols of $A$ we obtain a matrix which is a Latin square isotopic to $A$, and it satisfies the following: 
if in the first row, the symbol $x$ appears before $y$, with $x, y \in\{1,2\}$, and the symbol $z$ before $w$, with $z, w \in\{3,4\}$, then in all other rows we have

$$
x \text { before } y \text {, and } z \text { before } w,
$$

or

$$
y \text { before } x \text {, and } w \text { before } z \text {. }
$$

The isotopy relation is an equivalence relation in the class of Latin squares of order $n$. The equivalence classes are called isotopic classes. The aim of this paper is to proceed the work of [14], and study the restriction of the Bruhat order to an isotopic class of Latin squares.

The paper is organized as follows: In Section 2, we state conditions for two isotopic Latin squares, one obtained from the other by rows or/and columns interchanges be related by the Bruhat order. In Section 3, we make a similar study but when one of the Latin squares is obtained from the other by permutation of symbols. The cover relation between two isotopic Latin squares for the Bruhat order is the purpose of Section 4. In Section 5, we study isotopic orthogonal Latin squares that are related by the Bruhat order. Finally, in Section 6 we give some concluding remarks.

Let $(X, \preceq)$ be a finite partially order set and $a, b \in X$. If $a \neq b$ and $a \preceq b$ then we write $a \prec b$.

Note that if $A$ and $D$ are two Latin squares of order $n$ and $D$ is obtained from $A$ by interchanging different rows or columns or symbols then $A \neq D$. Consequently, $A \prec_{B} D$ or $D \prec_{B} A$ or $A$ and $D$ are not related by the Bruhat order.

As we are going to see, in a class of isotopic Latin squares the Bruhat order can, in some cases, be described using the majorization order on vectors. We recall the definition here:

Definition $1.1[5]$ Let $a=\left(a_{1}, \ldots, a_{n}\right)$, and $b=\left(b_{1}, \ldots, b_{n}\right)$ be two sequences of nonnegative integers with the same sum. We say that the sequence $b$ is majorized by the sequence $a$, and we write $b \preceq_{M} a$, if

$$
\sum_{l=1}^{t} b_{l} \leq \sum_{l=l}^{t} a_{l}, \quad t=1, \ldots, n .
$$

The sum of two integral vectors is the usual sum, and is denoted by $\oplus$.

\section{Rows or/and Columns Interchange}

In this section, we begin the study of isotopic Latin squares related by the Bruhat order. Here, the isotopic relation only allows the interchange of rows or columns. However, we will show a result involving the interchange of two rows and two columns, simultaneously. 
Theorem 2.1 Let $A=\left[a_{i, j}\right]$ be a Latin square of order $n$, and let $\pi \in S_{n} \backslash\{i d\}$. Let $G=\{i: 1 \leq i \leq n, \pi(i) \neq i\}$, and let $s_{1}<s_{2}<\ldots<s_{|G|}$ be the ordered elements of $G$. Then $A \prec_{B} P(\pi) A$ if and only if

$$
\oplus_{t=1}^{p}\left(a_{\pi^{-1}\left(s_{t}\right), 1}, \ldots, a_{\pi^{-1}\left(s_{t}\right), n}\right) \preceq_{M} \oplus_{t=1}^{p}\left(a_{s_{t}, 1}, \ldots, a_{s_{t}, n}\right),
$$

for $p=1, \ldots,|G|-1$.

Proof. If $l \in\{1, \ldots, n\}$ then the $\pi(l)$ th row of $P(\pi) A$ is the $l$ th row of $A$.

For all $r \in\{1, \ldots, n\}-\left\{s_{1}, s_{1}+1, \ldots, s_{|G|}-1\right\}$, and for $h \in\{1, \ldots, n\}$,

$$
\sigma_{r, h}(A)=\sigma_{r, h}(P(\pi) A) .
$$

Let $r \in\left\{s_{1}, s_{1}+1, \ldots, s_{|G|}-1\right\}$, and $h \in\{1, \ldots, n\}$. If $s_{p} \leq r<s_{p+1}$, with $1 \leq p \leq|G|-1$, then

$$
\sigma_{r, h}(P(\pi) A)=\sigma_{r, h}(A)+\sum_{t=1}^{p} \sum_{k=1}^{h} a_{\pi^{-1}\left(s_{t}\right), k}-\sum_{t=1}^{p} \sum_{k=1}^{h} a_{s_{t}, k} .
$$

So, if we assume that $A \prec_{B} P(\pi) A$, then $\sigma_{r, h}(A) \geq \sigma_{r, h}(P(\pi) A)$, for $r \in$ $\left\{s_{1}, s_{1}+1, \ldots, s_{|G|}-1\right\}$ and $h \in\{1, \ldots, n\}$. Consequently,

$$
\sum_{t=1}^{p} \sum_{k=1}^{h} a_{\pi^{-1}\left(s_{t}\right), k} \leq \sum_{t=1}^{p} \sum_{k=1}^{h} a_{s_{t}, k},
$$

for $h=1, \ldots, n$. Therefore,

$$
\oplus_{t=1}^{p}\left(a_{\pi^{-1}\left(s_{t}\right), 1}, \ldots, a_{\pi^{-1}\left(s_{t}\right), n}\right) \preceq_{M} \oplus_{t=1}^{p}\left(a_{s_{t}, 1}, \ldots, a_{s_{t}, n}\right),
$$

for $p=1, \ldots,|G|-1$. The converse is analogous.

The following corollary is an immediate consequence of last theorem when the permutation $\pi$ is a transposition.

Corollary 2.2 Let $A=\left[a_{i, j}\right]$ be a Latin square of order $n$, and let $i, j \in$ $\{1, \ldots, n\}, i<j$. Let $D$ be the Latin square obtained from $A$ by interchanging rows $i$ and $j$. Then $A \prec_{B} D$ if and only if $\left(a_{j, 1}, \ldots, a_{j, n}\right) \preceq_{M}\left(a_{i, 1}, \ldots, a_{i, n}\right)$.

Remark 2.3 1. Using last result we conclude that if rows $i$ and $j$ of $A$ are not related by the majorization order and $D$ is the matrix obtained from $A$ interchanging rows $i$ and $j$ then $A$ and $D$ are not related by the Bruhat order.

2. By Theorem 2.1, last remark can be generalized: So, if the sum of the rows $s_{1}, \ldots, s_{p}$ and the sum of the rows $s_{\pi(1)}, \ldots, s_{\pi(p)}$ of $A$, with $1 \leq p \leq$ $|G|-1$, are not related by the majorization order then $A$ and $P(\pi) A$ are not related by the Bruhat order. 
Another consequence of Theorem 2.1 involves a permutation that is a cycle of length three.

Corollary 2.4 Let $A=\left[a_{i, j}\right]$ be a Latin square of order $n$, with $n \geq 3$. Let $\pi=(i j k) \in S_{n}$ be a cycle of length three, with $1 \leq i<j<k \leq n$. If

$$
\left(a_{\pi^{-1}(i), 1}, \ldots, a_{\pi^{-1}(i), n}\right) \preceq_{M}\left(a_{i, 1}, \ldots, a_{i, n}\right),
$$

and

$$
\left(a_{\pi^{-1}(j), 1}, \ldots, a_{\pi^{-1}(j), n}\right) \preceq_{M}\left(a_{j, 1}, \ldots, a_{j, n}\right),
$$

then $A \prec_{B} P(\pi) A$.

Proof. Let $\theta \in S_{n}$ be the transposition $(i k)$. So, $\theta(i)=\pi^{-1}(i)$. Since $\left(a_{\pi^{-1}(i), 1}, \ldots, a_{\pi^{-1}(i), n}\right) \preceq_{M}\left(a_{i, 1}, \ldots, a_{i, n}\right)$, by Corollary $2.2, A \prec_{B} P(\theta) A$.

Since $\pi^{-1}(j)=i$ and the row $k$ of $P(\theta) A$ is the row $i$ of $A$, let $\xi$ be the transposition $(j k)$ of $S_{n}$. So, $\xi(j)=k$. Note that the row $j$ of $P(\theta) A$ is the row $j$ of $A$. Since

$$
\left((P(\theta) A)_{\xi^{-1}(j), 1}, \ldots,(P(\theta) A)_{\xi^{-1}(j), n}\right) \preceq_{M}\left((P(\theta) A)_{j, 1}, \ldots,(P(\theta) A)_{j, n}\right),
$$

by Corollary 2.2, $P(\theta) A \prec_{B} P(\xi) P(\theta) A$. Because $P(\xi) P(\theta)=P(\pi)$ we conclude the result.

The converse of last result does not hold as we can see in the next example.

Example 2.5 Let

$$
A=\left[\begin{array}{llll}
4 & 2 & 1 & 3 \\
2 & 4 & 3 & 1 \\
3 & 1 & 4 & 2 \\
1 & 3 & 2 & 4
\end{array}\right] \quad \text { and } \pi=(124) \in S_{n}
$$

Since

$$
\begin{gathered}
(1,3,2,4)=\left(a_{\pi^{-1}(1), 1}, \ldots, a_{\pi^{-1}(1), 4}\right) \preceq_{M}\left(a_{1,1}, \ldots, a_{1,4}\right)=(4,2,1,3), \\
\left(a_{1,1}, \ldots, a_{1,4}\right) \oplus\left(a_{2,1}, \ldots, a_{2,4}\right)=(4,2,1,3) \oplus(2,4,3,1)=(6,6,4,4), \\
\left(a_{\pi^{-1}(1), 1}, \ldots, a_{\pi^{-1}(1), 4}\right) \oplus\left(a_{\pi^{-1}(2), 1}, \ldots, a_{\pi^{-1}(2), 4}\right) \\
=(1,3,2,4) \oplus(4,2,1,3) \\
=(5,5,3,7),
\end{gathered}
$$

and

$$
(5,5,3,7) \preceq_{M}(6,6,4,4),
$$

by Theorem 2.1, $A \prec_{B}(P(\pi) A)$.

However, $(4,2,1,3)=\left(a_{\pi^{-1}(2), 1}, \ldots, a_{\pi^{-1}(2), 4}\right) \npreceq_{M}\left(a_{2,1}, \ldots, a_{2,4}\right)=(2,4,3,1)$. 
With similar arguments, we can prove analogous results to the Theorem 2.1 and its corollaries when the two isotopic Latin squares are obtained by interchanging columns instead of rows.

We finish this section with a result that involves a simultaneous interchange of two rows and two columns. When we interchange only rows (or only columns) in a fixed Latin square $A$, the Bruhat order is equivalent to the majorization order of the sum of the rows (of the columns) interchanged in $A$. This simplicity disappears when we interchange rows and columns simultaneously.

Theorem 2.6 Let $A=\left[a_{i, j}\right]$ be a Latin square of order $n$, and let $k, l, p, q \in$ $\{1, \ldots, n\}, k<l$, and $p<q$. Let $D$ be the Latin square obtained from $A$ by interchanging rows $k$ and $l$ and columns $p$ and $q$. Then $A \prec_{B} D$ if and only if the following three conditions hold:

1. If $t_{1} \in\{1, \ldots, n\}$ is such that $\sum_{u=1}^{t_{1}}\left(a_{u, p}-a_{u, q}\right)<0$, then $k \leq t_{1}<l$;

2. If $t_{2} \in\{1, \ldots, n\}$ is such that $\sum_{v=1}^{t_{2}}\left(a_{k, v}-a_{l, v}\right)<0$, then $p \leq t_{2}<q$;

3. If $k \leq t_{1}<l$ and $p \leq t_{2}<q$, then

$$
a_{k, p}-a_{l, q} \geq \sum_{\substack{u=1 \\ u \neq k}}^{t_{1}}\left(a_{u, q}-a_{u, p}\right)+\sum_{\substack{v=1 \\ v \neq p}}^{t_{2}}\left(a_{l, v}-a_{k, v}\right)
$$

Proof. Assume that $A \prec_{B} D$. Let $t_{1} \in\{1, \ldots, n\}$ such that

$$
\sum_{u=1}^{t_{1}}\left(a_{u, p}-a_{u, q}\right)<0
$$

and assume that $t_{1} \notin\{k, \ldots, l-1\}$. Then

$$
\sigma_{t_{1}, p}(A-D)=\sum_{u=1}^{t_{1}}\left(a_{u, p}-a_{u, q}\right)<0
$$

that is

$$
\sigma_{t_{1}, p}(A)<\sigma_{t_{1}, p}(D) \text {. }
$$

Hence we cannot have $A \prec_{B} D$. With similar arguments we prove that the condition 2. holds. Finally if $t_{1}, t_{2} \in\{1, \ldots, n\}, k \leq t_{1}<l, p \leq t_{2}<q$, since $\sigma_{t_{1}, t_{2}}(A-D) \geq 0$ then

$$
a_{k, p}-a_{l, q} \geq \sum_{\substack{u=1 \\ u \neq k}}^{t_{1}}\left(a_{u, q}-a_{u, p}\right)+\sum_{\substack{v=1 \\ v \neq p}}^{t_{2}}\left(a_{l, v}-a_{k, v}\right)
$$


and the condition 3 . holds.

Assume now that the three conditions hold. Let $i, j \in\{1, \ldots, n\}$.

Case 1: Assume that $i<k$. If $j<p$ or $j \geq q$, then

$$
\sigma_{i, j}(A)=\sigma_{i, j}(D),
$$

and if $p \leq j<q$, then by condition 1 .,

$$
\sigma_{i, j}(A-D)=\sum_{u=1}^{i}\left(a_{u, p}-a_{u, q}\right) \geq 0
$$

and then $\sigma_{i, j}(A) \geq \sigma_{i, j}(D)$.

Case 2: Assume that $k \leq i<l$. If $j<p$ or $j \geq q$, then by condition 2.,

$$
\sigma_{i, j}(A-D)=\sum_{v=1}^{j}\left(a_{k, v}-a_{l, v}\right) \geq 0,
$$

and then $\sigma_{i, j}(A) \geq \sigma_{i, j}(D)$. If $p \leq j<q$, then by condition 3 .,

$$
\sigma_{i, j}(A-D)=\sum_{\substack{v=1 \\ v \neq p}}^{j}\left(a_{k, v}-a_{l, v}\right)+\sum_{\substack{u=1 \\ u \neq k}}^{i}\left(a_{u, q}-a_{u, p}\right)+\left(a_{k, p}-a_{l, q}\right) \geq 0,
$$

and consequently, $\sigma_{i, j}(A) \geq \sigma_{i, j}(D)$.

Case 3: Assume that $i \geq l$. If $j<p$ or $j \geq q$, then

$$
\sigma_{i, j}(A)=\sigma_{i, j}(D),
$$

and if $p \leq j<q$, then by condition 1 .,

$$
\sigma_{i, j}(A-D)=\sum_{u=1}^{i}\left(a_{u, p}-a_{u, q}\right) \geq 0
$$

and consequently, $\sigma_{i, j}(A) \geq \sigma_{i, j}(D)$.

\section{Permutation of Symbols}

An alternative description of the Bruhat order between two Latin squares that are obtained one from the other by the permutation of symbols is the purpose of this section. In [14], the authors proved some results on this matter (see Section 2 of [14]). So, in this section the isotopic relation is only given by the permutation of symbols.

We begin with a crucial vector obtained from a Latin square that will be necessary to relate two isotopic Latin squares by the Bruhat order.

Given a matrix $A$, we denote by $A\left[\left\{i_{1}, \ldots, i_{t}\right\} \mid\left\{j_{1}, \ldots, j_{l}\right\}\right]$ the submatrix of $A$ that lies in rows $i_{1}, \ldots, i_{t}$, and columns $j_{1}, \ldots j_{l}$. 
Definition 3.1 Let $A=\left[a_{i, j}\right]$ be a Latin square of order $n$, and let $k \in$ $\{1, \ldots, n\}$. We denote by $S_{k}(A)$ the sequence of length $n^{2}$,

$$
S_{k}(A)=\left(s_{1,1}^{(k)}(A), \ldots, s_{1, n}^{(k)}(A), s_{2,1}^{(k)}(A), \ldots, s_{2, n}^{(k)}(A), \ldots, s_{n, 1}^{(k)}(A), \ldots, s_{n, n}^{(k)}(A)\right),
$$

where $s_{i, j}^{(k)}(A)$ is the number of entries equal to $k$ in the submatrix $A[\{1, \ldots, i\} \mid$ $\{1, \ldots, j\}]$.

Example 3.2 Let $A=\left[\begin{array}{lll}1 & 3 & 2 \\ 2 & 1 & 3 \\ 3 & 2 & 1\end{array}\right]$. Then

$$
\begin{aligned}
S_{2}(A)= & \left(s_{11}^{(2)}(A), s_{12}^{(2)}(A), s_{13}^{(2)}(A), s_{21}^{(2)}(A), s_{22}^{(2)}(A), s_{23}^{(2)}(A), s_{31}^{(2)}(A), s_{32}^{(2)}(A),\right. \\
= & \left.s_{33}^{(2)}(A)\right) \\
= & (0,0,1,1,1,2,1,2,3) .
\end{aligned}
$$

Note that, since $A$ is a Latin square of order $n$ and $k \in\{1, \ldots, n\}$ then $S_{k}(A)$ is not a zero vector. Moreover, all coordinates of $S_{k}(A)$ are nonnegative integers.

Recall that the sum of two integral vectors is the usual sum, denoted by $\oplus$. The product of an integer by an integral vector is the usual product. We denote by 0 the zero vector.

Lemma 3.3 Let $A$ be a Latin square of order $n$ and $\alpha \in S_{n}$. Then for $r, s \in$ $\{1, \ldots, n\}$,

$$
\sigma_{r, s}(\alpha(A))=\sigma_{r, s}(A)-\left(\sum_{i=1}^{n} s_{r s}^{(i)}(A)(i-\alpha(i))\right)
$$

Proof. Since $\alpha(A)$ is obtained from $A$ by interchanging the symbol $i$ by $\alpha(i)$ then the result follows.

Theorem 3.4 Let $A$ be a Latin square of order n, and let $\alpha \in S_{n} \backslash\{i d\}$. Then $A \prec_{B} \alpha(A)$ if and only if $\oplus_{i=1}^{n} S_{i}(A)(i-\alpha(i)) \geq 0$ by the entrywise order.

Proof. Assume that $A \prec_{B} \alpha(A)$, and assume that there exist $r, s \in\{1, \ldots, n\}$ such that $\sum_{i=1}^{n} s_{r s}^{(i)}(A)(i-\alpha(i))<0$. By Lemma 3.3,

$$
\sigma_{r, s}(\alpha(A))=\sigma_{r, s}(A)-\left(\sum_{i=1}^{n} s_{r s}^{(i)}(A)(i-\alpha(i))\right) .
$$

Consequently, $\sigma_{r, s}(\alpha(A))>\sigma_{r, s}(A)$, which is impossible. 
Conversely, assume that $\oplus_{i=1}^{n} S_{i}(A)(i-\alpha(i)) \geq 0$ by the entrywise order, and let $r, s \in\{1, \ldots, n\}$. By Lemma 3.3,

$$
\sigma_{r, s}(\alpha(A))=\sigma_{r, s}(A)-\left(\sum_{i=1}^{n} s_{r s}^{(i)}(A)(i-\alpha(i))\right) .
$$

So,

$$
\sigma_{r, s}(A) \geq \sigma_{r, s}(\alpha(A))
$$

Since $\alpha \in S_{n} \backslash\{i d\}$ then $A \neq \alpha(A)$ and $A \prec_{B} \alpha(A)$.

The following corollary is an immediate consequence of last theorem when the permutation $\alpha$ is a transposition.

Corollary 3.5 Let $A$ be a Latin square of order $n$, and let $k, l \in\{1, \ldots, n\}, k<$ $l$. Let $D$ be the Latin square obtained from $A$ by interchanging symbols $k$ and $l$. Then $A \prec_{B} D$ if and only if $S_{l}(A) \geq S_{k}(A)$ by the entrywise order.

Another consequence of Theorem 3.4 involves a permutation that is a composition of transpositions in the conditions of last corollary.

Corollary 3.6 Let $A$ be a Latin square of order $n$, and let $\alpha \in S_{n} \backslash\{i d\}$ such that $\alpha=\beta_{h} \circ \beta_{h-1} \circ \ldots \circ \beta_{1}$, where $\beta_{j}=\left(k_{j} l_{j}\right)$ is a transposition and $1 \leq\left(\beta_{j} \circ \beta_{j-1} \circ \ldots \circ \beta_{1}\right)\left(l_{j}\right)=k_{j}<l_{j} \leq n$, for $j=1, \ldots, h$. If

$$
S_{\left(\beta_{j-1} \circ \ldots \circ \beta_{1}\right)\left(l_{j}\right)}(A) \geq S_{\left(\beta_{j-1} \circ \ldots \circ \beta_{1}\right)\left(k_{j}\right)}(A),
$$

for $j=1, \ldots, h$, by the entrywise order then $A \prec_{B} \alpha(A)$.

Proof. Since $S_{l_{1}}(A) \geq S_{k_{1}}(A)$ and $k_{1}<l_{1}$, by Corollary 3.5 we have $A \prec_{B}$ $\beta_{1}(A)$. Because $S_{u}\left(\beta_{1}(A)\right)=S_{\beta_{1}(u)}(A)$, for $u=1, \ldots, n$, using the hypothesis and by Corollary 3.5 we have $\beta_{1}(A) \prec_{B}\left(\beta_{2} \circ \beta_{1}\right)(A)$. Consequently, $A \prec_{B}$ $\left(\beta_{2} \circ \beta_{1}\right)(A)$. Repeating these arguments, we get the result.

The converse of the last result does not hold as we can see in the next example.

Example 3.7 Let $A=\left[\begin{array}{llll}4 & 3 & 2 & 1 \\ 1 & 2 & 4 & 3 \\ 3 & 4 & 1 & 2 \\ 2 & 1 & 3 & 4\end{array}\right]$ and $\theta=(23)(14) \in S_{4}$.

So, $\theta(A)=\left[\begin{array}{llll}1 & 2 & 3 & 4 \\ 4 & 3 & 1 & 2 \\ 2 & 1 & 4 & 3 \\ 3 & 4 & 2 & 1\end{array}\right]$, and by calculations we get $A \prec_{B} \theta(A)$.

Moreover,

$$
S_{1}(A)=(0,0,0,1,1,1,1,2,1,1,2,3,1,2,3,4)
$$




$$
\begin{aligned}
& S_{2}(A)=(0,0,1,1,0,1,2,2,0,1,2,3,1,2,3,4), \\
& S_{3}(A)=(0,1,1,1,0,1,1,2,1,2,2,3,1,2,3,4), \\
& S_{4}(A)=(1,1,1,1,1,1,2,2,1,2,3,3,1,2,3,4) .
\end{aligned}
$$

Consequently, $S_{1}, S_{2}$ and $S_{3}$ are not related by the entrywise order, and $S_{4} \geq S_{1}$, $S_{4} \geq S_{2}, S_{4} \geq S_{3}$. To get $\alpha$ in the conditions of Corollary 3.5 we must have $\beta_{1}(14)$ or $\beta_{1}=(24)$ or $\beta_{1}=(34)$. This implies that the only way to change all integers between 1 and 4 using transpositions in the conditions of Corollary 3.5 is when $\alpha=\beta_{3} \circ \beta_{2} \circ \beta_{1}$, where $\beta_{1}=(34), \beta_{2}=(23)$ and $\beta_{3}=(12)$. So, $\alpha=(1234) \neq \theta$.

\section{The Cover Relation}

Let $(X, \preceq)$ be a finite partially order set and $a, b \in X$. We say that $b$ covers $a$ if $a \prec b$ and there is not $c \in X$ with $a \prec c \prec b$.

This section is about the cover relation for the Bruhat order on the class of isotopic Latin squares. In [14], Section 2, appeared some results about the cover relation between Latin squares related by the permutation of symbols. Now, using the permutation of two rows, the next result shows when the matrix obtained by this operation does not cover the initial matrix.

Theorem 4.1 Let $A=\left[a_{i, j}\right]$ be a Latin square of order $n$, and let $i, j \in$ $\{1, \ldots, n\}, i<j$, such that $\left(a_{j, 1}, \ldots, a_{j, n}\right) \preceq_{M}\left(a_{i, 1}, \ldots, a_{i, n}\right)$. Let $D$ be the Latin square obtained from $A$ by permuting the rows $i$ and $j$. If $D$ covers $A$ in the Bruhat order then for all $k, l$, with $i \leq k<l \leq j,\{i, j\} \neq\{k, l\}$ and $\left(a_{l, 1}, \ldots, a_{l, n}\right) \preceq_{M}\left(a_{k, 1}, \ldots, a_{k, n}\right)$, there is a $t \in\{1, \ldots, n\}$ such that

$$
\sum_{v=1}^{t}\left(a_{i, v}-a_{j, v}\right)<\sum_{v=1}^{t}\left(a_{k, v}-a_{l, v}\right) .
$$

Proof. Assume that $D$ covers $A$. Then $A \prec_{B} D$ and by Proposition 2.2, $\left(a_{j, 1}, \ldots, a_{j, n}\right) \preceq_{M}\left(a_{i, 1}, \ldots, a_{i, n}\right)$. Suppose that there are $k, l$ such that $i \leq k<$ $l \leq j,\{i, j\} \neq\{k, l\},\left(a_{l, 1}, \ldots, a_{l, n}\right) \preceq_{M}\left(a_{k, 1}, \ldots, a_{k, n}\right)$, and

$$
\sum_{v=1}^{t}\left(a_{i, v}-a_{j, v}\right) \geq \sum_{v=1}^{t}\left(a_{k, v}-a_{l, v}\right),
$$

for all $t \in\{1, \ldots, n\}$.

Let $C=\left[c_{i, j}\right]$ be the Latin square obtained from $A$ by permuting rows $k$ and $l$. Since $\left(a_{l, 1}, \ldots, a_{l, n}\right) \preceq_{M}\left(a_{k, 1}, \ldots, a_{k, n}\right)$, by Proposition $2.2, A \prec_{B} C$. If we prove that $C \prec_{B} D$, as $D$ covers $A$ we get a contradiction. First note that $C \neq D$ because $\{i, j\} \neq\{k, l\}$ and we are dealing with Latin squares.

As $D=\left[d_{i j}\right]$ results from $A$ by permuting rows $i$ and $j$, and $C$ results from $A$ by permuting rows $k$ and $l$, with $i \leq k<l \leq j$, we have, for $r \in$ 
$\{1, \ldots, n\}-\{i, k, l, j\}$ and $t \in\{1, \ldots, n\}, a_{r, t}=c_{r, t}=d_{r, t}$. Therefore, for $r \in\{1, \ldots, i-1\}$,

$$
\sigma_{r, t}(A)=\sigma_{r, t}(C)=\sigma_{r, t}(D), \text { for } t=1, \ldots, n .
$$

If $r \in\{i, \ldots, k-1\}$, as $c_{r, t}=a_{r, t}$, for $t=1, \ldots, n$ we have

$$
\sigma_{r, t}(C)=\sigma_{r, t}(A) \geq \sigma_{r, t}(D), \text { for } t=1, \ldots, n .
$$

Let $r \in\{k, \ldots, l-1\}$. Since

$$
\sigma_{r, t}(A-C)=\sum_{v=1}^{t}\left(a_{k, v}-c_{k, v}\right)=\sum_{v=1}^{t}\left(a_{k, v}-a_{l, v}\right),
$$

and

$$
\sigma_{r, t}(A-D)=\sum_{v=1}^{t}\left(a_{k, v}-c_{k, v}\right)=\sum_{v=1}^{t}\left(a_{i, v}-a_{j, v}\right),
$$

we conclude that

$$
\sigma_{r, t}(A-D) \geq \sigma_{r, t}(A-C), \text { for } t=1, \ldots, n .
$$

Then, for $r \in\{k, \ldots, l-1\}$ and $t \in\{1, \ldots, n\}$ we get

$$
\sigma_{r, t}(C) \geq \sigma_{r, t}(D), \text { for } t=1, \ldots, n .
$$

If $r \geq l$, then

$$
\sigma_{r, t}(C)=\sigma_{r, t}(A) \geq \sigma_{r, t}(D), \quad \text { for } t=1, \ldots, n .
$$

Then $A \prec_{B} C \prec_{B} D$, which is a contradiction.

The converse of last theorem does not hold as we can see in the following example.

\section{Example 4.2 Let}

$$
A=\left[\begin{array}{lllll}
1 & 2 & 5 & 4 & 3 \\
5 & 3 & 4 & 2 & 1 \\
4 & 5 & 1 & 3 & 2 \\
3 & 1 & 2 & 5 & 4 \\
2 & 4 & 3 & 1 & 5
\end{array}\right]
$$

Then

$$
\Sigma_{A}=\left[\begin{array}{ccccc}
1 & 3 & 8 & 12 & 15 \\
6 & 11 & 20 & 26 & 30 \\
10 & 20 & 30 & 39 & 45 \\
13 & 24 & 36 & 50 & 60 \\
15 & 30 & 45 & 60 & 75
\end{array}\right]
$$


Since rows 2 and 5 of $A$ verify

$$
(2,4,3,1,5) \preceq_{M}(5,3,4,2,1),
$$

by Proposition 2.2, the matrix $D$ obtained from $A$ interchanging rows 2 and 5 satisfies $A \prec_{B} D$. Observe that

$$
D=\left[\begin{array}{lllll}
1 & 2 & 5 & 4 & 3 \\
2 & 4 & 3 & 1 & 5 \\
4 & 5 & 1 & 3 & 2 \\
3 & 1 & 2 & 5 & 4 \\
5 & 3 & 4 & 2 & 1
\end{array}\right]
$$

and

$$
\Sigma_{D}=\left[\begin{array}{ccccc}
1 & 3 & 8 & 12 & 15 \\
3 & 9 & 17 & 22 & 30 \\
7 & 18 & 27 & 35 & 45 \\
10 & 22 & 33 & 46 & 60 \\
15 & 30 & 45 & 60 & 75
\end{array}\right]
$$

Let $C$ be the Latin square obtained by applying the permutations (2435), (345) and (2453) to the symbols, rows and columns of $A$, respectively. Then $A, C, D$ are isotopic Latin squares,

$$
C=\left[\begin{array}{lllll}
1 & 2 & 5 & 4 & 3 \\
3 & 5 & 4 & 1 & 2 \\
4 & 3 & 1 & 2 & 5 \\
5 & 1 & 2 & 3 & 4 \\
2 & 4 & 3 & 5 & 1
\end{array}\right] \text { and } \Sigma_{C}=\left[\begin{array}{ccccc}
1 & 3 & 8 & 12 & 15 \\
4 & 11 & 20 & 25 & 30 \\
8 & 18 & 28 & 35 & 45 \\
13 & 24 & 36 & 46 & 60 \\
15 & 30 & 45 & 60 & 75
\end{array}\right]
$$

So, $A \prec_{B} C \prec_{B} D$ and $D$ does not cover $A$.

On the other hand, denoting by $r_{i}$ the ith row of $A=\left[a_{i j}\right]$, for $i=1,2,3,4$, we have $r_{3} \swarrow_{M} r_{2}, r_{4} \preceq_{M} r_{2}$, and

$$
\begin{gathered}
\left(a_{21}-a_{51}\right)+\left(a_{22}-a_{52}\right)=2<4=\left(a_{21}-a_{41}\right)+\left(a_{22}-a_{42}\right), \\
r_{4} \preceq_{M} r_{3} \text { and }\left(a_{21}-a_{51}\right)+\left(a_{22}-a_{52}\right)=2<5=\left(a_{31}-a_{41}\right)+\left(a_{32}-a_{42}\right), \\
r_{5} \preceq_{M} r_{3} \text { and }\left(a_{21}-a_{51}\right)+\left(a_{22}-a_{52}\right)=2<3=\left(a_{31}-a_{51}\right)+\left(a_{32}-a_{52}\right), \\
r_{5} \npreceq_{M} r_{4} .
\end{gathered}
$$

So, $A$ and $D$ verify the conditions of last proposition.

An easy consequence of Theorem 4.1 is the next corollary.

Corollary 4.3 Let $A=\left[a_{i, j}\right]$ be a Latin square of order $n$, and let $i, s, j \in$ $\{1, \ldots, n\}$, with $i<s<j$, such that $\left(a_{j, 1}, \ldots, a_{j, n}\right) \preceq_{M}\left(a_{s, 1}, \ldots, a_{s, n}\right) \preceq_{M}$ $\left(a_{i, 1}, \ldots, a_{i, n}\right)$. Let $D$ be the Latin square obtained from $A$ by permuting rows $i$ and $j$. Then $D$ does not cover $A$.

With the same arguments we can prove similar results for the interchange of columns. 


\section{Orthogonal isotopic Latin squares}

As we mentioned in the Introduction, Ryser's conjectured that if $A$ is a Latin square of odd order then $A$ has, at least, one transversal. Since this conjecture remains unproven in general, the purpose of this section is to describe Latin squares of order $3^{k}$, with $k \geq 1$, that have transversals. For this we describe two Latin squares of order $3^{k}$ that are orthogonal and related by the Bruhat order.

We begin this section with the notation of some matrices that are needed in the next results:

Let $J_{h}$ be the matrix of order $h$ whose all entries are equal to one, $0_{h}$ be the matrix of order $h$ whose all entries are equal to zero, and $I_{h}$ be the identity matrix of order $h$.

For $p$ a positive integer and $p \geq 2$, let

$$
R_{p}=\left[\begin{array}{c|c|c}
\left(2 \times 3^{p-1}\right) J_{3^{p-1}} & 0_{3^{p-1}} & 3^{p-1} J_{3^{p-1}} \\
\hline 3^{p-1} J_{3^{p-1}} & \left(2 \times 3^{p-1}\right) J_{3^{p-1}} & 0_{3^{p-1}} \\
\hline 0_{3^{p-1}} & 3^{p-1} J_{3^{p-1}} & \left(2 \times 3^{p-1}\right) J_{3^{p-1}}
\end{array}\right],
$$

and

$$
T_{p}=\left[\begin{array}{c|c|c}
0_{3^{p-1}} & \left(2 \times 3^{p-1}\right) J_{3^{p-1}} & 3^{p-1} J_{3^{p-1}} \\
\hline\left(2 \times 3^{p-1}\right) J_{3^{p-1}} & 3^{p-1} J_{3^{p-1}} & 0_{3^{p-1}} \\
\hline 3^{p-1} J_{3^{p-1}} & 0_{3^{p-1}} & \left(2 \times 3^{p-1}\right) J_{3^{p-1}}
\end{array}\right] .
$$

Remark 5.1 1. The matrix $T_{p}$ is obtained from $R_{p}$ interchanging columns.

In fact,

$R_{p}\left[\begin{array}{c|c|c}0_{3^{p-1}} & I_{3^{p-1}} & 0_{3^{p-1}} \\ \hline I_{3^{p-1}} & 0_{3^{p-1}} & 0_{3^{p-1}} \\ \hline 0_{3^{p-1}} & 0_{3^{p-1}} & I_{3^{p-1}}\end{array}\right]=T_{p}$.

2. Let $p \geq 2$ and $\rho$ be a permutation of $S_{3^{p}}$ such that for each $a \in\left\{0,3^{p-1}, 2 \times\right.$ $\left.3^{p-1}\right\}$ there is $b_{a} \in\left\{0,3^{p-1}, 2 \times 3^{p-1}\right\}$ satisfying the condition:

$$
\text { if } i \in\left\{a+1, \ldots, a+3^{p-1}\right\} \text { then } \rho^{-1}(i) \in\left\{b_{a}+1, \ldots, b_{a}+3^{p-1}\right\} .
$$

Then, $T_{p} P(\rho)=T_{p}$. 
Lemma 5.2 Let $p$ be a positive integer with $p \geq 2$. Let $R_{p}$ and $T_{p}$ be the matrices described before. Then $R_{p} \prec_{B} T_{p}$.

Proof. Let $i, j \in\left\{1, \ldots, 3^{p}\right\}$. To simplify we will write $c$ instead of $3^{p-1}$. Then,

$$
\sigma_{i j}\left(R_{p}\right)= \begin{cases}2 c i j & \text { if } 1 \leq i, j \leq c \\ 2 c^{2} i & \text { if } 1 \leq i \leq c, c+1 \leq j \leq 2 c \\ 2 c^{2} i+c(j-2 c) i & \text { if } 1 \leq i \leq c, 2 c+1 \leq j \leq 3 c \\ 2 c^{2} j+c j(i-c) & \text { if } c+1 \leq i \leq 2 c, 1 \leq j \leq c \\ 2 c^{3}+c^{2}(i-c)+2 c(j-c)(i-c) & \text { if } c+1 \leq i, j \leq 2 c \\ 2 c^{3}+c^{2}(i-c)+ & \\ 2 c^{2}(i-c)+c^{2}(j-2 c) & \text { if } c+1 \leq i \leq 2 c<j \leq 3 c \\ 2 c^{2} j+c^{2} j & \text { if } i>2 c+1,1 \leq j \leq c \\ 2 c^{3}+c^{3}+2 c^{2}(j-c)+ & \text { if } i>2 c+1, \\ c(j-c)(i-2 c) & c+1 \leq j \leq 2 c \\ 4 c^{3}+c^{3}+c^{2}(j-2 c)+ & \\ c^{2}(i-2 c)+2 c(j-2 c)(i-2 c) & \text { if } i, j>2 c+1\end{cases}
$$

and

$$
\sigma_{i j}\left(T_{p}\right)= \begin{cases}0 & \text { if } 1 \leq i, j \leq c \\ 2 c(j-c) i & \text { if } 1 \leq i \leq c, c+1 \leq j \leq 2 c \\ 2 c^{2} i+c(j-2 c) i & \text { if } 1 \leq i \leq c, 2 c+1 \leq j \leq 3 c \\ 2 c(i-c) j & \text { if } c+1 \leq i \leq 2 c, 1 \leq j \leq c \\ 2 c^{2}(j-c)+2 c^{2}(i-c)+c(j-c)(i-c) & \text { if } c+1 \leq i, j \leq 2 c \\ 2 c^{3}+2 c^{2}(i-c)+ & \\ c^{2}(i-c)+c^{2}(j-2 c) & \text { if } c+1 \leq i \leq 2 c<j \leq 3 c \\ 2 c^{2} j+c(i-2 c) j & \text { if } i>2 c+1,1 \leq j \leq c \\ 2 c^{3}+c^{2}(i-2 c)+2 c^{2}(j-c)+ & \text { if } i>2 c+1, \\ c^{2}(j-c) & c+1 \leq j \leq 2 c \\ 4 c^{3}+c^{3}+c^{2}(j-2 c)+c^{2}(i-2 c)+ & \\ 2 c(j-2 c)(i-2 c) & \text { if } i, j>2 c+1\end{cases}
$$


So, if we prove that $\sigma_{i j}\left(R_{p}\right) \geq \sigma_{i j}\left(T_{p}\right)$, for $c+1 \leq i, j \leq 2 c$ and for $i \geq 2 c, c+1 \leq$ $j \leq 2 c$ then we will conclude that $R_{p} \prec_{B} T_{p}$.

Case 1 Suppose that $c+1 \leq i, j \leq 2 c$.

Let $a=i-c$ and $b=j-c$. Then $1 \leq a, b \leq c$ and

$\sigma_{i j}\left(R_{p}\right)-\sigma_{i j}\left(T_{p}\right) \geq 0$ if and only if

$$
2 c(c-(a+b))+a(c+b) \geq 0 .
$$

If $a+b \leq c$ then $2 c(c-(a+b))+a(c+b) \geq 0$.

Suppose that $a+b=c+r$, with $1 \leq r \leq c$. If $a<r$ then $c+r=a+b<r+b$.

Consequently, $b>c$, which is impossible. So, $a \geq r$.

Therefore,

$$
2 c(c-(a+b))+a(c+b)=(a-r)(2 c-a) \geq 0 .
$$

Case 2 Suppose that $i \geq 2 c, c+1 \leq j \leq 2 c$.

Let $a=i-2 c$ and $b=j-c$. Then $1 \leq a, b \leq c$ and

$\sigma_{i j}\left(R_{p}\right)-\sigma_{i j}\left(T_{p}\right) \geq 0$ if and only if

$$
(a-c)(b-c) \geq 0 .
$$

In [14], Section 4, we can see the Hasse diagram of the class of Latin squares of order 3 for $\preceq_{B}$

where

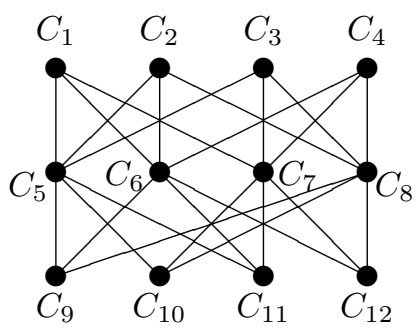

$$
\begin{aligned}
& C_{1}=\left[\begin{array}{lll}
1 & 2 & 3 \\
2 & 3 & 1 \\
3 & 1 & 2
\end{array}\right], C_{2}=\left[\begin{array}{lll}
1 & 2 & 3 \\
3 & 1 & 2 \\
2 & 3 & 1
\end{array}\right], C_{3}=\left[\begin{array}{lll}
1 & 3 & 2 \\
2 & 1 & 3 \\
3 & 2 & 1
\end{array}\right], C_{4}=\left[\begin{array}{lll}
2 & 1 & 3 \\
1 & 3 & 2 \\
3 & 2 & 1
\end{array}\right], \\
& C_{5}=\left[\begin{array}{lll}
1 & 3 & 2 \\
3 & 2 & 1 \\
2 & 1 & 3
\end{array}\right], C_{6}=\left[\begin{array}{lll}
2 & 1 & 3 \\
3 & 2 & 1 \\
1 & 3 & 2
\end{array}\right], C_{7}=\left[\begin{array}{lll}
2 & 3 & 1 \\
1 & 2 & 3 \\
3 & 1 & 2
\end{array}\right], C_{8}=\left[\begin{array}{lll}
3 & 1 & 2 \\
1 & 2 & 3 \\
2 & 3 & 1
\end{array}\right], \\
& C_{9}=\left[\begin{array}{lll}
3 & 1 & 2 \\
2 & 3 & 1 \\
1 & 2 & 3
\end{array}\right], C_{10}=\left[\begin{array}{lll}
3 & 2 & 1 \\
1 & 3 & 2 \\
2 & 1 & 3
\end{array}\right], C_{11}=\left[\begin{array}{lll}
2 & 3 & 1 \\
3 & 1 & 2 \\
1 & 2 & 3
\end{array}\right], C_{12}=\left[\begin{array}{lll}
3 & 2 & 1 \\
2 & 1 & 3 \\
1 & 3 & 2
\end{array}\right] .
\end{aligned}
$$

So, by the Hasse diagram and using the matrices, we get 8 hypothesis: 
- $C_{9} \prec_{B} C_{5}$, they are isotopic (interchange columns 1 and 2) and orthogonal.

- $C_{10} \prec_{B} C_{8}$, they are isotopic (interchange columns 2,3 ) and orthogonal.

- $C_{11} \prec_{B} C_{6}$, they are isotopic (interchange columns 2,3 ) and orthogonal.

- $C_{12} \prec_{B} C_{7}$, they are isotopic (interchange columns 1,2 ) and orthogonal.

- $C_{9} \prec_{B} C_{4}$, they are isotopic (interchange columns 1 and 3) and orthogonal.

- $C_{10} \prec_{B} C_{1}$, they are isotopic (interchange columns 1,3 ) and orthogonal.

- $C_{11} \prec_{B} C_{3}$, they are isotopic (interchange columns 1,3 ) and orthogonal.

- $C_{12} \prec_{B} C_{2}$, they are isotopic (interchange columns 1,3 ) and orthogonal.

Denote by $D_{1}$ and $E_{1}$ the two Latin squares of order 3 which are the matrices that appear in one of the 8 hypothesis described before, with $D_{1} \prec_{B} E_{1}$.

Recursively, for $p \geq 2$, let

and

$$
D_{p}=R_{p}+\left[\begin{array}{c|c|c}
D_{p-1} & D_{p-1} & D_{p-1} \\
\hline D_{p-1} & D_{p-1} & D_{p-1} \\
\hline D_{p-1} & D_{p-1} & D_{p-1}
\end{array}\right],
$$

$$
E_{p}=T_{p}+\left[\begin{array}{c|c|c}
E_{p-1} & E_{p-1} & E_{p-1} \\
\hline E_{p-1} & E_{p-1} & E_{p-1} \\
\hline E_{p-1} & E_{p-1} & E_{p-1}
\end{array}\right] .
$$

Now, we can prove the main result of this section.

Theorem 5.3 Let $p$ be a positive integer and let $D_{p}$ and $E_{p}$ be the matrices described before. Then $D_{p}$ and $E_{p}$ are isotopic orthogonal Latin squares and $D_{p} \prec_{B} E_{p}$. Moreover, $E_{p}$ is obtained from $D_{p}$ only interchanging columns.

Proof. The proof is by induction in $p$.

For $p=1$, since $D_{1}$ and $E_{1}$ are in the conditions of one of last items, with $D_{1} \prec_{B} E_{1}$, the result follows.

Suppose that for some positive integer $p-1$, with $p-1 \geq 1, D_{p-1}$ and $E_{p-1}$ are isotopic orthogonal Latin squares and $D_{p-1} \prec_{B} E_{p-1}$. By construction, $D_{p}$ and $E_{p}$ are Latin squares.

Using Remark 5.1,

$$
D_{p}\left[\begin{array}{c|c|c}
0_{3^{p-1}} & I_{3^{p-1}} & 0_{3^{p-1}} \\
\hline I_{3^{p-1}} & 0_{3^{p-1}} & 0_{3^{p-1}} \\
\hline 0_{3^{p-1}} & 0_{3^{p-1}} & I_{3^{p-1}}
\end{array}\right]=T_{p}+\left[\begin{array}{c|c|c}
D_{p-1} & D_{p-1} & D_{p-1} \\
\hline D_{p-1} & D_{p-1} & D_{p-1} \\
\hline D_{p-1} & D_{p-1} & D_{p-1}
\end{array}\right] .
$$


Since $E_{p-1}$ is obtained from $D_{p-1}$ interchanging columns, by Remark 5.1, $D_{p}$ and $E_{p}$ are isotopic using only the interchange of columns.

On the other hand, since $D_{p-1}$ and $E_{p-1}$ are orthogonal matrices and, $R_{p}$ and $T_{p}$ are orthogonal by blocks then $D_{p}$ and $E_{p}$ are orthogonal matrices.

Because $D_{p-1} \prec_{B} E_{p-1}$, using Lemma 5.2 we conclude that $D_{p} \prec_{B} E_{p}$. Therefore, the result follows.

\section{Conclusions}

We have studied the Bruhat order on the class of isotopic Latin squares. In particular, we have established necessary and sufficient conditions for two Latin squares, of the same order and isotopic, to be related by this order.More precisely, in this study the isotopic relation only was allowed by the interchange of rows or of columns or of symbols.

The cover relation in the Bruhat order was also studied but only in the case when the two Latin squares were related by a permutation of two rows or of two columns.

We have finished this paper studying the conjecture of Ryser "All Latin squares of odd order have, at least, one transversal". So, we have presented Latin squares of order $3^{k}$, where $k$ is an integer greater than or equal to one, that are orthogonal and related by the Bruhat order.

It is an interesting problem to describe all Latin squares of odd order that are orthogonal and related by the Bruhat order.

\section{Acknowledgements}

We thank the anonymous referees for many useful suggestions.

\section{References}

[1] K. Balasubramanian, On transversals in latin squares, Linear Algebra Appl., 131 (1990) 125-129.

[2] D. Best and I.M. Wanless, Parity of transversals of Latin Squares, arXiv.org $>$ math $>$ arXiv.1912.11230.

[3] J.N. Bray, Q. Cai, P.J. Cameron, P.Spiga and H. Zhang, The Hall-Page conjecture, and Synchronization for affine and diagonal groups, Journal of Algebra, 545 (2020) 27-42.

[4] R.A. Brualdi and S.-G. Hwang, A Bruhat order for the class of $(0,1)$ matrices with row sum vector $R$ and column sum vector $S$, Electronic Journal of Linear Algebra, 12 (2004) 6-16. 
[5] R.A. Brualdi, Combinatorial Matrix Classes, Encyclopedia of Mathematics and its Applications, vol. 108, Cambridge University Press, Cambridge (2006).

[6] R.A. Brualdi and L. Deaett, More on the Bruhat order for $(0,1)$-matrices, Linear Algebra Appl., 421 (2007) 219-232.

[7] R.A. Brualdi and G. Dahl, Doubly stochastic matrices and the Bruhat order, Czechoslovak Mathematical Journal, 66 (141) (2016) 681-700.

[8] R.A. Brualdi and E. Fritscher, Bruhat order of tournaments, Linear Algebra Appl., 458 (2014) 261-279.

[9] R.A. Brualdi and M. W.Schroeder, Alternating sign matrices and their Bruhat order, Discrete Mathematics, 340, Issue 8 (2017) 1996-2019.

[10] R.A. Brualdi, R. Fernandes and S. Furtado, On the Bruhat order of labeled graphs, Discrete Applied Mathematics, 258 (2019) 49-64.

[11] A. Conflitti, C.M. Fonseca and R. Mamede, The maximal lenght of a chain in the Bruhat order for a class of binary matrices, Linear Algebra Appl., 436 (2012), 753-757.

[12] A. Conflitti, C.M. da Fonseca and R. Mamede, On the largest size of an antichain in the Bruhat order for $\mathcal{A}(2 k, k)$, Order, 30 (2013), 255-260

[13] H.F. Cruz, R. Fernandes and S. Furtado, Minimal matrices in the Bruhat order for symmetric (0,1)-matrices, Linear Algebra Appl., 530 (2017) 160184 .

[14] R. Fernandes, H.F. da Cruz and D. Salomão, Latin Squares and their Bruhat order, Contributions to Discrete Mathematics, 15 (2020) 102-120.

[15] R. Fernandes, H.F. da Cruz and D. Salomão, Classes of (0,1)-matrices where the Bruhat order and the Secondary Bruhat order coincide, Order. https://doi.org/10.1007/s11083-019-09500-8

[16] R. Fernandes and S. Furtado, Extremal matrices for the Bruhat-graph order, Linear and Multilinear Algebra, https://doi.org/10.1080/03081087.2020.1749540

[17] R. Fernandes, H.F. da Cruz and D. Salomão, On a conjecture concerning the Bruhat order, Linear Algebra Appl., 600 (2020) 82-95.

[18] M. Ghebleh, On maximum chains in the Bruhat order of $\mathcal{A}(n, 2)$, Linear Algebra Appl., 446 (2014), 377-387.

[19] M. Ghebleh, Antichains on (0,1)-matrices through inversions, Linear Algebra Appl.,458 (2014), 503-511 
[20] B.D. McKay, J.C. McLeod, and I.M. Wanless, The number of transversals in a latin square, Designs, Codes and Cryptography, 40 (2006) 269-284.

[21] H.J. Ryser. Neuere probleme der kombinatorik, Vorträge über Kombinatorik, Oberwolfach (1967) 69-91. 